\title{
Some anomalies of the curved bar problem in classical elasticity
}

\author{
J.R. BARBER \\ Department of Mechanical Engineering and Applied Mechanics, University of Michigan, \\ Ann Arbor, MI 48109-2125, USA
}

Received 10 March 1989

\begin{abstract}
The classical formulation of the 'homogeneous' problem of a curved bar loaded only by an end force involves the assumption of an appropriate stress function with four arbitrary constants and the determination of these constants from the boundary conditions. Since there are five boundary conditions, four on the curved edge and one at the end, the solution is only possible because the coefficient matrix of the resulting algebraic equations is singular. This in turn means that certain inhomogeneous problems in which the curved edges are loaded by sinusoidally varying tractions cannot be solved using apparently appropriate stress functions.

Additional stress functions which resolve this difficulty are introduced and an example problem is solved, which exhibits qualitatively different behavior from that in more general cases of loading. The problem is then reconsidered as a limiting case of sinusoidal loading of arbitrary wavelength. It is shown that the solution of the latter problem appears to become unbounded as the special case is approached, but that when the end conditions have been correctly satisfied by superposing an appropriate multiple of the end-loaded solution, the limit can be approached regularly and the correct special solution is recovered. The limiting process reveals a general procedure for determining the additional stress functions required for the special case.

Similar relationships between homogeneous and inhomogeneous solutions for other common geometries are discussed.
\end{abstract}

\section{The curved bar problem}

Figure 1 shows a classical problem in two-dimensional linear elasticity. A curved bar, defined in polar coordinates by $a<r<b, 0<\theta<\pi / 2$ is built in at the end $\theta=\pi / 2$ and loaded by a shear force $F$ at $\theta=0$, the curved boundaries $r=a, b$ being traction-free.

Most textbooks follow Timoshenko and Goodier [1] in arguing semiinversely that the bending moment will vary with $\sin (\theta)$ and hence look for an Airy stress function of the form $\phi=f(r) \sin (\theta)$, the appropriate function ${ }^{1}$ being

$$
\phi=\left\{A r^{3}+B r^{-1}+C r \log (r)+D r\right\} \sin (\theta) .
$$

\footnotetext{
1 The expression $\log (r)$ is strictly not meaningful if $r$ is a linear dimension. However, it is always found in such cases that the final expressions for the stresses can be grouped so as to avoid this difficulty (see for example [1], Art 29). Alternatively, we can interpret the quantities $r, a, b$ as denoting the dimensionless ratios $r / L, a / L, b / L$ between the actual dimensions and a suitable length, $L$, in which case the problem does not arise. Similar considerations apply to the expression $r^{\lambda}$ in Section 3 below.
} 


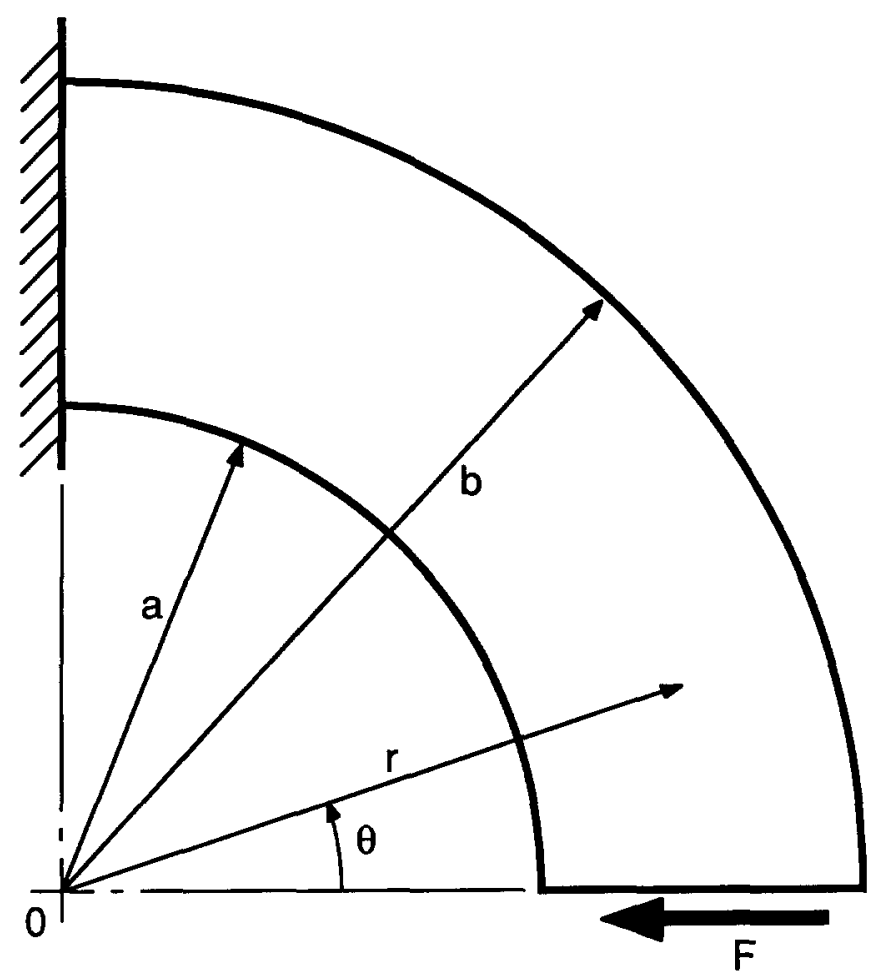

Fig. 1. The 'homogeneous' problem of a curved bar with an end load.

We note, incidentally, that one of the terms in (1) is trivial in the sense that it defines a null stress field so that it might be more rational to seek a function $\phi$ such that the stresses vary with $\sin (\theta)$, the appropriate function being

$$
\phi=\left\{A r^{3}+B r^{-1}+C r \log (r)\right\} \sin (\theta)+D r \theta \cos (\theta) .
$$

However, this minor modification does not significantly affect the argument.

The stress components derived from (2) are

$$
\begin{aligned}
& \sigma_{r r}=\left\{2 A r-2 B r^{-3}+C r^{-1}-2 D r^{-1}\right\} \sin (\theta), \\
& \sigma_{r \theta}=\left\{-2 A r+2 B r^{-3}-C r^{-1}\right\} \cos (\theta), \\
& \sigma_{\theta \theta}=\left\{6 A r+2 B r^{-3}+C r^{-1}\right\} \sin (\theta),
\end{aligned}
$$

and we can now determine the constants $A, B, C, D$ by imposing the boundary conditions

$$
\begin{gathered}
\sigma_{r r}=0, \quad r=a, b, \\
\sigma_{r \theta}=0, \quad r=a, b,
\end{gathered}
$$


and

$$
\int_{a}^{b} \sigma_{r \theta} \mathrm{d} r=F, \quad \theta=0
$$

Conditions (4) lead to the set of equations

$$
\begin{aligned}
& 2 A a-2 B / a^{3}+C / a-2 D / a=0 \\
& 2 A b-2 B / b^{3}+C / b-2 D / b=0, \\
& 2 A a-2 B / a^{3}+C / a=0 \\
& 2 A b-2 B / b^{3}+C / b=0
\end{aligned}
$$

and the final solution is obtained as

$$
A=F / 2 N, \quad B=-F a^{2} b^{2} / 2 N, \quad C=-F\left(a^{2}+b^{2}\right) / N, \quad D=0,
$$

where

$$
N=\left(a^{2}-b^{2}\right)+\left(a^{2}+b^{2}\right) \log (b / a)
$$

The more intelligent graduate students are wont to inquire at this point how it is that we are able to satisfy five conditions with only four independent solutions. Clearly the four linear algebraic equations (6) are not linearly independent, though none of the textbook treatments of this problem remark on the fact, nor do they address the question 'Is it just luck that this is the case and that the problem therefore has a solution of the assumed form, or should we have expected the equations derived from conditions (4) not to be linearly independent?'

\section{The inhomogeneous problem}

The fact that the equations (6) are not linearly independent implies that the matrix of coefficients is singular and this in turn means that we shall get into difficulties with the corresponding inhomogeneous problem in which one or more of the boundary conditions (4) is replaced by one involving a traction of the appropriate form. 


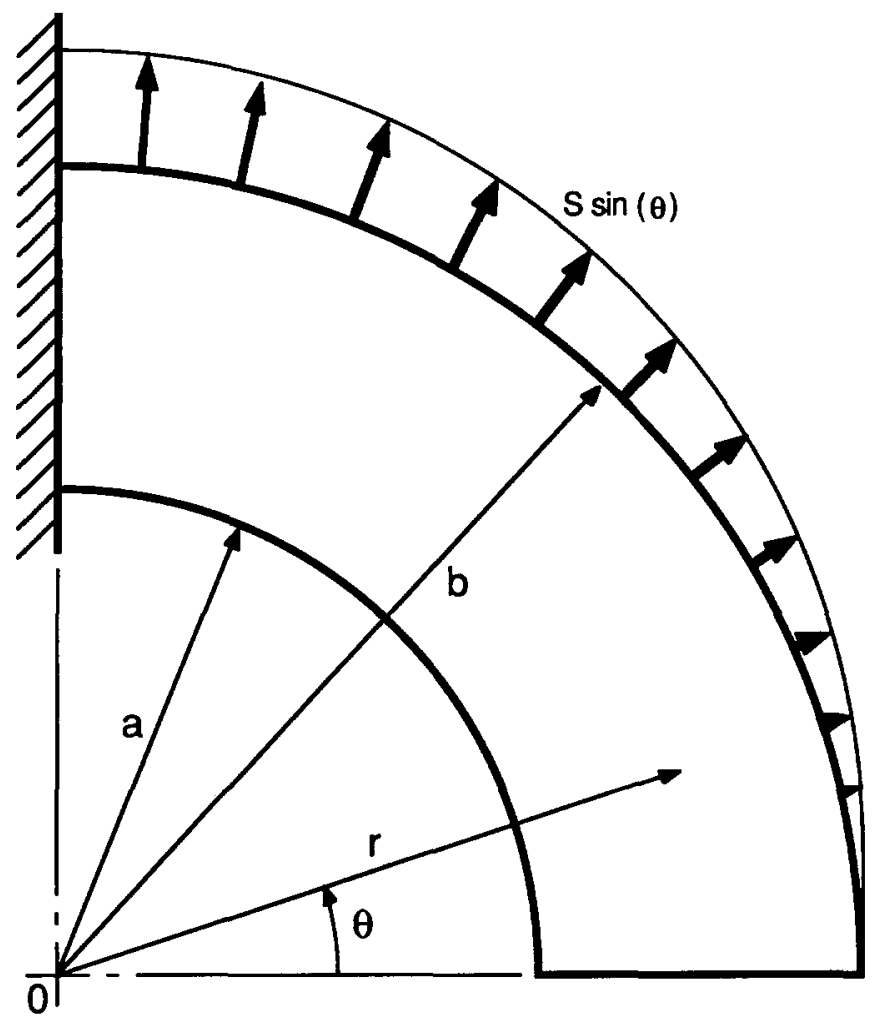

Fig. 2. The inhomogeneous problem; sinusoidally varying function, but no end load.

For example, the problem of Fig. 2 has the boundary conditions

$$
\begin{array}{ll}
\sigma_{r r}=0, & r=a, \\
\sigma_{r r}=S \sin (\theta), & r=b, \\
\sigma_{r \theta}=0, & r=a, b,
\end{array}
$$

and

$$
\int_{a}^{b} \sigma_{r \theta} \mathrm{d} r=0, \quad \theta=0
$$

The form of the only non-zero traction in (9) leads us to expect stresses of the form of equation (3), but the substitution of (3) into (9) will give a set of equations identical to (6) except that the right hand side of the second equation is replaced by $S$. 
Since the matrix of coefficients of these equations is singular, the equations cannot be solved and hence there does not exist a solution to the problem of Fig. 2 of the assumed form.

In such cases, we must seek a special solution in which the dependence of the stress field on $\theta$ differs from that in the boundary conditions. Such special solutions are well-known for other geometries. For example, the wedge loaded by uniform tractions on its faces has a solution with stresses independent of distance from the apex except for two special values of the wedge angle. One of these is the case of the half-plane (a wedge of 180 degrees) loaded by a uniform shear traction on one side of the origin, for which we use the special stress function $\left\{r^{2} \log (r) \cos (2 \theta)-r^{2} \theta \sin (2 \theta)\right\}$, leading to logarithmic variation of stresses with $r$.

For the curved bar problem, we can generate a suitable special solution by expressing the original function (2) in complex variable form and then multiplying it by $\log (z)$. Thus, (2) consists of a linear combination of the terms $\operatorname{Im}\left\{\bar{z} z^{2} ; z^{-1} ; z \log (z) ; \bar{z} \log (z)\right\}$, so we generate a new biharmonic function from the terms $\operatorname{Im}\left\{\bar{z} z^{2} \log (z) ; z^{-1} \log (z) ; z(\log (z))^{2} ; \bar{z}(\log (z))^{2}\right\}$ which can be written in the form

$$
\begin{aligned}
\phi= & A^{\prime}\left\{r^{3} \log (r) \sin (\theta)+r^{3} \theta \sin (\theta)\right\}+B^{\prime}\{\theta \cos (\theta)-\log (r) \sin (\theta)\} / r \\
& +C^{\prime} r \log (r) \theta \cos (\theta)+D^{\prime}\left\{r \log ^{2}(r) \sin (\theta)-r \theta^{2} \sin (\theta)\right\}
\end{aligned}
$$

The corresponding stress components are

$$
\begin{aligned}
\sigma_{r r}= & \left\{2 A^{\prime} r-2 B^{\prime} r^{-3}+C^{\prime} r^{-1}-4 D^{\prime} r^{-1}\right\} \theta \cos (\theta) \\
& +\left\{2 A^{\prime} r \log (r)-A^{\prime} r+2 B^{\prime} r^{-3} \log (r)-3 B^{\prime} r^{-3}\right. \\
& \left.-2 C^{\prime} r^{-1} \log (r)+2 D^{\prime} r^{-1} \log (r)-2 D^{\prime} r^{-1}\right\} \sin (\theta), \\
\sigma_{r \theta}= & \left\{2 A^{\prime} r-2 B^{\prime} r^{-3}+C^{\prime} r^{-1}\right\} \theta \sin (\theta)+\left\{-2 A^{\prime} r \log (r)-3 A^{\prime} r\right. \\
& \left.-2 B^{\prime} r^{-3} \log (r)+3 B^{\prime} r^{-3}-C^{\prime} r^{-1}-2 D^{\prime} r^{-1} \log (r)\right\} \cos (\theta), \\
\sigma_{\theta \theta}= & \left\{6 A^{\prime} r+2 B^{\prime} r^{-3}+C^{\prime} r^{-1}\right\} \theta \cos (\theta)+\left\{6 A^{\prime} r \log (r)+5 A^{\prime} r\right. \\
& \left.-2 B^{\prime} r^{-3} \log (r)+3 B^{\prime} r^{-3}+2 D^{\prime} r^{-1} \log (r)+2 D^{\prime} r^{-1}\right\} \sin (\theta) .
\end{aligned}
$$

Notice in particular that these expressions contain some terms of the required form for the problem of Fig. 2, but they also contain terms with multipliers of the form $\theta \cos (\theta), \theta \sin (\theta)$, which are inappropriate. We there- 
fore get four homogeneous equations for the coefficients $A^{\prime}, B^{\prime}, C^{\prime}, D^{\prime}$ from the requirement that these inappropriate terms should vanish in the components $\sigma_{r r}, \sigma_{r \theta}$ on the boundaries $r=a, b$. i.e.

$$
\begin{aligned}
& 2 A^{\prime} a-2 B^{\prime} \mid a^{3}+C^{\prime} / a-4 D^{\prime} / a=0 \\
& 2 A^{\prime} b-2 B^{\prime} / b^{3}+C^{\prime} \mid b-4 D^{\prime} / b=0 \\
& 2 A^{\prime} a-2 B^{\prime} / a^{3}+C^{\prime} / a=0 \\
& 2 A^{\prime} b-2 B^{\prime}\left|b^{3}+C^{\prime}\right| b=0
\end{aligned}
$$

Clearly these equations are identical with (6) and are not linearly independent. There is therefore a non-trivial solution to (15), which leaves us with a stress function which can supplement that of equation (2) to make the problem well-posed. From here on, the solution is algebraically complicated but routine. Adding the two stress functions $(2,11)$, we obtain a function with 8 unknown constants, which is required to satisfy 9 boundary conditions, comprising (i) equations (15), (ii) equations (6) modified to include $\sin (\theta)$, $\cos (\theta)$ terms from equations $(12,13)$ respectively and (iii) equation (10). Since two of equations (15) are not independent, the system reduces to a set of 8 equations for 8 constants, whose solution is

$$
\begin{aligned}
A^{\prime}= & S b / 4 N ; \quad B^{\prime}=-S a^{2} b^{3} / 4 N ; \quad C^{\prime}=-S b\left(a^{2}+b^{2}\right) / 2 N ; \quad D^{\prime}=0 ; \\
A= & \frac{S b}{8 N^{2}}\left[2\left(b^{2}-a^{2}\right)+\left(3 a^{2}+b^{2}\right) \log (b)+\left(3 b^{2}+a^{2}\right) \log (a)\right. \\
& \left.-2\left\{a^{2} \log (a)+b^{2} \log (b)\right\} \log (b / a)\right] \\
B= & \frac{S a^{2} b^{3}}{8 N^{2}}\left[-2\left(b^{2}-a^{2}\right)+\left(3 b^{2}+a^{2}\right) \log (b)-\left(3 a^{2}+b^{2}\right) \log (a)\right. \\
& \left.-2\left\{a^{2} \log (b)+b^{2} \log (a)\right\} \log (b / a)\right] \\
C= & \frac{S b}{4 N^{2}}\left[2\left(b^{4}+a^{4}\right) \log (b / a)-\left(b^{4}-a^{4}\right)\right] \\
D= & \frac{S b}{4 N}\left[\left(b^{2}-a^{2}\right)+2\left(b^{2}+a^{2}\right) \log (a)\right]
\end{aligned}
$$


Of course, it is not fortuitous that the stress function (11) leads to a set of equations (15) identical with (6). When we differentiate the function $f(z, \bar{z}) \log (z)$ by parts to determine the stresses, the extra multiplier $\log (z)$ is only preserved in those terms where it is not differentiated and hence in which all the differential operations are performed on $f(z, \bar{z})$. But these operations on $f(z, \bar{z})$ are precisely those leading to the stresses in the original solution and hence to equations (6). The reader will notice a parallel here with the procedure for determining the general solution of a differential equation with repeated differential multipliers.

\section{The near-singular problem}

Suppose we next consider a more general version of the problem of Fig. 2 in which the inhomogeneous boundary condition (9) is replaced by

$$
\sigma_{r r}=S \sin (\lambda \theta), \quad r=b,
$$

where $\lambda$ is a constant. In the special case where $\lambda=1$, this problem reduces to that of Section 2. For all other values (excluding $\lambda=0$ ), we can use the stress function

$$
\phi=\left\{A r^{\lambda+2}+B r^{\lambda}+C r^{-\lambda}+D r^{-\lambda+2}\right\} \sin (\lambda \theta)
$$

with stress components

$$
\begin{aligned}
\sigma_{r r}= & -\left\{A(\lambda-2)(\lambda+1) r^{\lambda}+B \lambda(\lambda-1) r^{\lambda-2}+C \lambda(\lambda+1) r^{-\lambda-2}\right. \\
& \left.+D(\lambda+2)(\lambda-1) r^{-\lambda}\right\} \sin (\lambda \theta), \\
\sigma_{r \theta}= & \left\{-A \lambda(\lambda+1) r^{\lambda}-B \lambda(\lambda-1) r^{\lambda-2}+C \lambda(\lambda+1) r^{-\lambda-2}\right. \\
& \left.+D \lambda(\lambda-1) r^{-\lambda}\right\} \cos (\lambda \theta), \\
\sigma_{\theta \theta}= & \left\{A(\lambda+1)(\lambda+2) r^{\lambda}+B \lambda(\lambda-1) r^{\lambda-2}+C \lambda(\lambda+1) r^{-\lambda-2}\right. \\
& \left.+D(\lambda-1)(\lambda-2) r^{-\lambda}\right\} \sin (\lambda \theta) .
\end{aligned}
$$


The boundary conditions on the curved edges lead to the four equations

$$
\begin{aligned}
& A(\lambda-2)(\lambda+1) a^{\lambda}+B \lambda(\lambda-1) a^{\lambda-2}+C \lambda(\lambda+1) a^{-\lambda-2} \\
& \quad+D(\lambda+2)(\lambda-1) a^{-\lambda}=0 \\
& A(\lambda-2)(\lambda+1) b^{\lambda}+B \lambda(\lambda-1) b^{\lambda-2}+C \lambda(\lambda+1) b^{-\lambda-2} \\
& \quad+D(\lambda+2)(\lambda-1) b^{-\lambda}=-S \\
& -A \lambda(\lambda+1) a^{\lambda}-B \lambda(\lambda-1) a^{\lambda-2}+C \lambda(\lambda+1) a^{-\lambda-2}+D \lambda(\lambda-1) a^{-\lambda}=0 \\
& -A \lambda(\lambda+1) b^{\lambda}-B \lambda(\lambda-1) b^{\lambda-2}+C \lambda(\lambda+1) b^{-\lambda-2}+D \lambda(\lambda-1) b^{-\lambda}=0
\end{aligned}
$$

which have the solution

$$
\begin{aligned}
& A=\frac{S b^{\lambda}}{2(\lambda+1) M}\left[(\lambda+1) f(\lambda)-\lambda b^{2} f(\lambda-1)\right], \\
& B=\frac{-S b^{\lambda}}{2(\lambda-1) M}\left[\lambda f(\lambda+1)-(\lambda-1) b^{2} f(\lambda)\right], \\
& C=\frac{-S a^{2 \lambda} b^{\lambda+2}}{2(\lambda+1) M}\left[\lambda b^{2 \lambda-2} f(1)+f(\lambda)\right], \\
& D=\frac{S^{2 \lambda-2} b^{\lambda}}{2(\lambda-1) M}\left[\lambda b^{2 \lambda} f(1)+a^{2} f(\lambda)\right],
\end{aligned}
$$

where

$$
\begin{aligned}
& M=\lambda^{2} f(\lambda-1) f(\lambda+1)-\left(\lambda^{2}-1\right) f(\lambda)^{2}, \\
& f(p)=b^{2 p}-a^{2 p}
\end{aligned}
$$

On casual inspection, it seems that this problem behaves rather remarkably as $\lambda$ passes through unity. The stress field is sinusoidal for all $\lambda \neq 1$ and increases without limit as $\lambda$ approaches unity from either side, since $M=0$ when $\lambda=1$. (Notice that an additional singularity is introduced through the factor $(\lambda-1)$ in the denominator of the coefficients $B, D$.) However, when $\lambda$ is exactly equal to unity, the problem has the bounded solution derived in Section 2, in which the stress field is not sinusoidal. 
However, we shall demonstrate that solution is not as discontinuous as it looks. The stress field obtained by substituting (21) into (18) is not a complete solution to the problem since the end condition (10) is not satisfied. The solution from (18) will generally involve a non-zero force on the end, given by

$$
\begin{aligned}
F & =\int_{a}^{b} \sigma_{r \theta} \mathrm{d} r \\
& =-\lambda\left[A f\left(\frac{\lambda+1}{2}\right)+B f\left(\frac{\lambda-1}{2}\right)+C f\left(\frac{-\lambda-1}{2}\right)+D f\left(\frac{-\lambda+1}{2}\right)\right] .
\end{aligned}
$$

To restore the no-traction condition on the end (in the weak sense of zero force resultant), we must subtract the solution of the homogeneous problem of Section 1, with $F$ given by (24). The final solution therefore involves the superposition of the stress functions (2) and (18). As $\lambda$ approaches unity, both components of the solution increase without limit, since (24) contains the singular terms (21) but they also tend to assume the same form since, for example, $r^{2+\lambda} \sin (\lambda \theta)$ approaches $r^{3} \sin (\theta)$. In the limit, the corresponding term takes the form

$$
\lim _{\lambda \rightarrow 1} \frac{A_{1}(\lambda) r^{2+\lambda} \sin (\lambda \theta)-A_{2}(\lambda) r^{3} \sin (\theta)}{M(\lambda)},
$$

where

$$
\begin{aligned}
& A_{1}(\lambda)=\frac{S b^{\lambda}}{2(\lambda+1)}\left[(\lambda+1) f(\lambda)-\lambda b^{2} f(\lambda-1),\right. \\
& A_{2}(\lambda)=\frac{F(\lambda) M(\lambda)}{2 N} .
\end{aligned}
$$

Setting $\lambda=1$ in equations $(26,27)$, we find

$$
A_{1}(1)=A_{2}(1)=\frac{S b^{2}\left(b^{2}-a^{2}\right)}{2} \text {. }
$$

The zero in $M(\lambda)$ is therefore cancelled and (25) has a bounded limit which can be recovered by using L'Hopital's rule. The resulting algebra is routine but lengthy and will not be reproduced here, but it is clear that in addition to a term proportional to $r^{3} \sin (\theta)$, the differentiation of the numerator in (25) 
will generate a term proportional to

$$
\frac{\partial}{\partial \lambda} r^{2+\lambda} \sin (\lambda \theta)=r^{2+\lambda}\{\log (r) \sin (\lambda \theta)+\theta \cos (\lambda \theta)\}
$$

which in the limit $\lambda=1$ reduces to $r^{3}\{\log (r) \sin (\theta)+\theta \cos (\theta)\}$. The coefficient of this term will be

$$
\begin{aligned}
A^{\prime} & =\frac{A_{1}(1)}{M^{\prime}(1)} \\
& =\frac{S b}{4 N}
\end{aligned}
$$

agreeing with the corresponding coefficient $A^{\prime}$ in the special solution of Section 2 (see equations (16)).

A similar limiting process yields the form and coefficients of the remaining 7 stress functions in the solution of Section 2. In particular, we note that the coefficients $B, D$ in equations (21) have a second singular term in the denominator, and hence L'Hopital's rule has to be applied twice, leading to stress functions of the form of the last two terms in equation (11).

Thus, we find that the solution to the more general problem of equation (17) includes that of Section 2 as a limiting case, and there are no values of $\lambda$ for which the stress field is singular. The limiting process also shows us a more general way of obtaining the special stress functions required for the problem of Section 2 . We simply take the stress function (18), which degenerates when $\lambda=1$, and differentiate it with respect to the parameter $\lambda$. Since the last two terms of (2) are already of this differential form, a second differentiation is required to generate the last two terms of (11).

\section{Some general considerations}

Similar examples could be found for other geometries in the two-dimensional theory of elasticity. To fix ideas, suppose we consider the body $a<\xi<b$, $c<\eta<d$ in the general system of curvilinear coordinates $\xi, \eta$. In general we might anticipate a class of separated variable solutions of the form

$$
\phi=f_{\lambda}(\xi) g_{\lambda}(\eta)
$$

where $\lambda$ is a parameter. An important physical problem is that in which the boundaries $\eta=c, d$ are traction free and the tractions on the remaining 
boundaries have a non-zero force or moment resultant. In such cases, we can generally use dimensional and/or equilibrium arguments to determine the form of the stress variation in the $\xi$ direction and hence the appropriate function $f_{\lambda}^{*}(\xi)$. The biharmonic equation will then reduce to a fourth order ordinary differential equation for $g_{\lambda}^{*}(\eta)$, with four linearly independent solutions. Enforcement of the traction free boundary conditions will give a set of four homogeneous equations for the unknown multipliers of these four solutions.

If the solution is to be possible, the equations must have a non-trivial solution, implying that the matrix of coefficients is singular. It therefore follows that the corresponding inhomogeneous problem cannot be solved by the stress function of equation (31) if the tractions on $\eta=c, d$ vary with $f_{\lambda}^{*}(\xi)$ - i.e. in the same way as the stresses in the homogeneous (end loaded) problem. However, special stress functions appropriate to this limiting case can be obtained by differentiating (31) with respect to the parameter $\lambda$.

The special solution for $\lambda=\lambda^{*}$ is not qualitatively different from that at more general values of $\lambda$, but appears as a regular limit once appropriate boundary conditions are imposed on the edges $\xi=a, b$.

Other simple examples in the two-dimensional theory of elasticity include the curved bar in bending [1, Art 29] and the wedge with traction free faces [1, Art 38]. Similar arguments can also be applied to axisymmetric problems - e.g. to problems of the cone with traction free surfaces.

The inhomogeneous problem of the wedge of semi-angle $\alpha$ loaded by tractions proportional to $r^{\lambda}$ on the faces $\theta= \pm \alpha$ can generally be solved using the stress function

$$
\phi=r^{\lambda+2}\{A \cos (\lambda \theta)+B \sin (\lambda \theta)+C \cos [(\lambda+2) \theta]+D \sin [(\lambda+2) \theta]\} .
$$

However, it is well-known that the wedge has solutions with traction free boundary conditions for the cases $\lambda=-1$ (the Flamant solution), $\lambda=-2$ (the concentrated moment solution), and for the Williams eigenvalue solutions [1, Art 46]. In each of these cases, the solution of the related inhomogeneous problem requires special stress functions which are obtained from (32) by differentiation with respect to $\lambda$.

For the special case of the half-plane $(\alpha=\pi / 2), \lambda=0$ and $\lambda=1$ are both eigenvalues. The homogeneous solutions in this case correspond to a state of uniaxial tension and bending described by $\sigma_{x x}=S_{0}+S_{1} y, \sigma_{x y}=\sigma_{y y}=0$ in the Cartesian coordinate system in which the half-plane is defined by $y>0$. The inhomogeneous problem for this case involves uniform or linearly varying tractions on the wedge faces (and hence on one or both halves of the surface of the half-space). The corresponding special solutions are obtained by differentiating (32) with respect to $\lambda$ and include the well-known functions 
$\left\{r^{2} \log (r) \sin (2 \theta)+r^{2} \theta \cos (2 \theta)\right\},\left\{r^{3} \log (r) \sin (3 \theta)+r^{3} \theta \cos (3 \theta)\right\}$, etc. (see [1] Chapter 4, problems $17,19,20,21)$.

As in the curved beam problem, the inhomogeneous wedge problem appears to exhibit a discontinuity in behavior at $\alpha=\pi / 2$, the stresses varying logarithmically with $r$ in the special case, but being uniform (or linear) for wedge semi-angles very close to $\pi / 2$. We anticipate that this difficulty could be resolved in the same way, by superposing a solution chosen to satisfy a suitable boundary condition on a remote boundary for the wedge (at $r=R$, where $R$ is in some sense large). The appropriate solution to superpose is that one of the Williams asymptotic solutions [2] whose variation with $r$ approaches $r^{0}$ (constant) or $r^{1}$ (linear) as $\alpha \rightarrow \pi / 2$. However, in this case, the multiplier on the added homogeneous solution cannot be determined from an equilibrium condition.

\section{Conclusions}

1. Whenever we find a classical solution in which there are two traction-free boundaries, we can formulate a related inhomogeneous problem for which the equation system resulting from these boundary conditions will be singular.

2. Special solutions for these cases can be obtained by parametric differentiation of more general solutions for the same geometry.

3. Alternatively, the special case can be recovered as a limit as the parameter tends to its eigenvalue, provided that the remaining boundary conditions are satisfied in an appropriate sense (e.g. in the weak sense of force resultants) before the limit is taken.

\section{References}

1. S.P. Timoshenko and J.N. Goodier, Theory of Elasticity, McGraw-Hill, New York, 3rd edn. (1970).

2. M.L. Williams, Stress singularities from various boundary conditions in angular corners of plates in extension, J. Appl. Mech. 19 (1952) 526-528. 